\title{
ASSOCIAÇÃO ENTRE SISTEMA DE INCENTIVOS GERENCIAIS E PRÁTICAS DE CONTABILIDADE GERENCIAL
}

\author{
ASSOCIATION BETWEEN MANAGEMENT INCENTIVE SYSTEMS AND MANAGEMENT \\ ACCOUNTING PRACTICES
}

ASOCIACIÓN ENTRE SISTEMA DE INCENTIVOS GERENCIALES Y PRÁCTICAS DE CONTABILIDAD GERENCIAL

\section{RESUMO}

O princípio da controlabilidade prevê que os gestores deveriam ser avaliados com base em fatores controláveis. Consequentemente, os incentivos gerenciais estariam relacionados a práticas de contabilidade gerencial capazes de evitar que os gestores sejam responsabilizados por resultados financeiros além do controle gerencial, tais como análise por centro de responsabilidade, custo padrão, preço de transferência, orçamento e avaliação de desempenho. Este artigo desenvolve um estudo de campo para investigar se há relação entre a presença de incentivos gerenciais e as práticas de contabilidade gerencial associadas ao princípio da controlabilidade. Entrevistas in loco foram realizadas para a coleta de dados em nível organizacional e testes estatísticos não paramétricos foram utilizados para a análise dos dados. Entre as práticas de contabilidade gerencial examinadas, os resultados sugerem que apenas orçamento anual, análise por centro de responsabilidade e avaliação de desempenho estão associados à presença de sistemas de incentivos nas empresas entrevistadas.

PALAVRAS-CHAVE Controle gerencial, práticas de contabilidade gerencial, avaliação de desempenho, sistemas de incentivo gerencial, controlabilidade.

Andson Braga de Aguiar abraga@usp.br

Professor da Faculdade de Economia, Administração e Contabilidade, Universidade de São Paulo - São Paulo - SP, Brasil

Aridelmo J. C. Teixeira aridelmo@fucape.br

Professor da Fucape Business School - Vitoria - ES, Brasil

Valcemiro Nossa valcemiro@fucape.br

Professor da Fucape Business School - Vitoria - ES, Brasil

Rosimeire Pimentel Gonzaga ropgonzaga@gmail.com

Doutoranda em Controladoria e Contabilidade pela Faculdade de Economia, Administração e Contabilidade, Universidade de São Paulo - São Paulo - SP, Brasil

\begin{abstract}
The controllability principle posits that managers should be evaluated based on controllable factors. Consequently, managerial incentives might be related to managerial accounting practices able to avoid that managers will be accountable for financial results beyond management control, such as analysis by responsibility center, standard-cost, transfer pricing, budgeting and performance evaluation. This paper develops a field research to investigate whether there is a relation between the presence of managerial incentives and managerial accounting practices associated with the controllability principle. On-site interviews were conducted to collect data at the organizational level and nonparametric statistical tests were used for data analysis. Among the managerial accounting practices examined, the results suggest that only the annual budget, analysis by responsibility center and performance evaluation are associated with the presence of incentive systems in the sampled firms.

Keywords Managerial control, management accounting practices, performance evaluation, management incentive systems, controllability.

Resumen El principio de la controlabilidad prevé que los gestores deberían ser evaluados con base en factores controlables. Consecuentemente, los incentivos gerenciales estarían relacionados a prácticas de contabilidad gerencial capaces de evitar que los gestores sean responsabilizados por resultados financieros más allá del control gerencial, tales como análisis por centro de responsabilidad, costo estándar, precio de transferencia, presupuesto y evaluación de desempeño. Este artículo desarrolla un estudio de campo para investigar si existe relación entre la presencia de incentivos gerenciales y las prácticas de contabilidad gerencial asociadas al principio de la controlabilidad. Para la recolección de datos en ámbito organizacional se realizaron entrevistas in situ y para el análisis de los datos se aplicaron tests estadísticos no paramétricos. Entre las prácticas de contabilidad gerencial examinadas, los resultados sugieren que solamente el presupuesto anual, el análisis por centro de responsabilidad y la evaluación de desempeño están asociados a la presencia de sistemas de incentivo en las empresas entrevistadas.
\end{abstract}

Palabras clave Control gerencial, prácticas de contabilidad gerencial, evaluación de desempeño, sistemas de incentivo gerencial, controlabilidad. 


\section{INTRODUÇÃo}

Sistemas de incentivo gerencial são percebidos como importante ferramenta de controle gerencial que cumpre um papel motivacional de induzir o comportamento dos gestores em direção aos objetivos organizacionais e, desse modo, garantir um determinado nível de desempenho (MERCHANT e VAN DER STEDE, 2003; BAILEY, BROWN, COCCO, 1998). Especificamente, incentivos monetários provocariam um aumento do esforço gerencial, que, por sua vez, afetaria positivamente o desempenho organizacional (BONNER e SPRINKLE, 2002). Sistemas de incentivo gerencial teriam, portanto, o efeito potencial de alinhar os interesses de agentes com aversão ao risco e ao esforço com aqueles dos acionistas (INDJEJIKIAN e NANDA, 1999).

A literatura contábil indica que uma das questões básicas associadas ao desenvolvimento de sistemas de incentivo gerencial se refere ao grau de controle que os gestores têm sobre as ações pelas quais eles são avaliados (FITZGERALD, 2007). Segundo o princípio da controlabilidade, os gestores deveriam ser responsabilizados apenas pelos resultados financeiros associados a atividades as quais eles possam influenciar ou controlar. Como consequência da aplicação desse princípio, os gestores deveriam ser avaliados e remunerados somente com base nas medidas de desempenho as quais eles também possam influenciar ou controlar (ANTLE e DEMSKI, 1988; HIRST, 1983).

Assim, a literatura contábil tem identificado uma série de práticas gerenciais que pode ser útil para viabilizar a aplicação do princípio da controlabilidade e para estruturar sistemas de incentivo gerencial. Tais práticas incluiriam: centros de responsabilidade, custo padrão, preço de transferência, orçamento e sistemas de avaliação de desempenho (ATKINSON e outros, 1995; MERCHANT e VAN DER STEDE, 2003). Sistemas de incentivo gerencial representariam, assim, o elo final de um processo de avaliação de desempenho (BANKER e DATAR, 1989; FITZGERALD, 2007). Nesse processo, os incentivos dependeriam de um determinado nível de desempenho alcançado em comparação com alguma meta preestabelecida, orçamentária ou não, baseada em padrões ou não. Por sua vez, essa avaliação de desempenho indicaria o grau de sucesso de um gestor na alocação de recursos a ele disponibilizados, podendo ser utilizados, para tanto, preços de transferência e orçamentos. Por fim, o processo de avaliação de desempenho incluiria apenas, ou principalmente, aquelas dimensões de desempenho que refletem as áreas de responsabilidade dos gestores, conforme definido pelo princípio da controlabilidade e representado, estruturalmente, pelos centros de responsabilidade.

Seria provável, portanto, que a presença de incentivos gerenciais somente ocorresse em empresas que possuem essas práticas gerenciais, em todo ou em parte. Espera-se, assim, que empresas que oferecem incentivos gerenciais aos gestores apresentem, ainda que em parte, estruturas descentralizadas organizadas em centros de responsabilidades, sistemas de custeio padrão, sistemas de alocação de recursos com base em preços de transferência e em orçamento, além de sistemas de avaliação de desempenho.

Este estudo pretende justamente investigar se, de fato, há relação entre a presença de sistemas de incentivo gerencial e as práticas de contabilidade gerencial associadas ao princípio da controlabilidade. Para tanto, desenvolve-se uma pesquisa de campo com uma amostra de empresas situadas no estado do Espírito Santo.

O objetivo deste estudo é, portanto, verificar se empresas que afirmam oferecer sistemas de incentivo gerencial, de fato, utilizam práticas de contabilidade gerencial - centros de responsabilidade, custo padrão, preço de transferência, orçamento e sistemas de avaliação de desempenho - que viabilizam a aplicação do princípio da controlabilidade.

Como principal contribuição para a pesquisa em contabilidade gerencial, temos a de identificar as práticas de contabilidade gerencial que são mais relevantes para que um sistema de incentivo gerencial possa ser estruturado, dado o princípio da controlabilidade. A utilização de tais práticas gerenciais poderia ser percebida como uma pré-condição para que uma empresa pudesse oferecer incentivos gerenciais aos seus gestores.

O restante do artigo é organizado dentro de quatro seções. Na seção 2, apresenta-se uma visão geral do princípio de controlabilidade, além de serem discutidas as práticas de contabilidade gerencial que estariam associadas com a aplicação de tal princípio. Na seção 3, são destacados os aspectos metodológicos empregados na pesquisa. A seção 4 apresenta os principais resultados da pesquisa de campo. Por fim, na seção 5, são elaboradas as conclusões do estudo, em que se discutem as principais implicações dos resultados alcançados, além de sugestões para futuras pesquisas. 


\section{PRINCÍPIO DA CONTROLABILIDADE - VISÃO GERAL}

O princípio da controlabilidade prevê que um gestor deve ser avaliado de acordo com o que ele pode controlar (ANTLE e DEMSKI, 1988; HIRST, 1983). Os livros-textos em contabilidade têm geralmente enfatizado o papel do princípio da controlabilidade como base para o desenho de relatórios contábeis gerenciais e de sistemas de avaliação de desempenho. Atkinson e outros (1995) destacam que a principal aplicação desse princípio é que um gestor não poderia ser responsável pelas receitas, pelos custos e pelos investimentos que não sejam de seu controle. Merchant e Van der Stede (2003) mencionam que uma das condições para que o controle financeiro possa ser efetivo é que os gestores cujos comportamentos estão sendo controlados sejam capazes de influenciar, de maneira significativa, os resultados esperados.

O atendimento ao princípio da controlabilidade busca evitar que gestores assumam o risco de fatores incontroláveis, o que poderia afetar negativamente o seu nível de motivação, além de induzir a comportamentos disfuncionais, tais como manipulação de resultados, criação de reservas e miopia gerencial (HIRST, 1983; MERCHANT e VAN DER STEDE, 2003). Além disso, fazer com que os gestores assumam riscos de fatores incontroláveis pode resultar em um custo excessivamente alto para as empresas (MERCHANT e VAN DER STEDE, 2003). No entanto, o que pode acontecer na prática é que os gestores sejam responsáveis apenas por uma quantidade não muito grande de fatores incontroláveis (MERCHANT e VAN DER STEDE, 2003).

Embora represente um tema relevante para o desenho de sistemas de contabilidade gerencial, poucos estudos apresentam uma definição formal do termo "controlabilidade". Lambert (2001) afirma que uma variável pode ser percebida como controlável na medida em que as ações gerenciais influenciam a distribuição de probabilidades daquela variável. Antle e Demski (1988) mencionam que essa definição direciona o foco de controle para os inputs (por exemplo, esforço gerencial, decisões de investimento e alocação de recursos) ao invés de outputs (por exemplo, medidas de desempenho). Nesse sentido, para controlar custos, receitas ou qualquer outra medida de desempenho, que representam os outputs, os gestores deveriam ser capazes de afetá-los por meio de seus esforços e de suas decisões gerenciais (ANTLE e DEMSKI, 1988).

Entretanto, Antle e Demski (1988) apresentam um modelo de agência em que sugerem que a noção de controlabilidade seria, de fato, representada pela capacidade dos gestores de afetarem a probabilidade condicional dos outputs, isto é, das medidas de desempenho. Simons (2007) afirma, então, que um indicador de desempenho deveria ser considerado como controlável em sentido relativo, o que poderia ocorrer por meio, por exemplo, da observação do desempenho alcançado por concorrentes em termos do mesmo indicador. Como consequência, qualquer informação de desempenho, ainda que imprecisa ou não perfeitamente controlável, seria útil para os propósitos de incentivo gerencial (BANKER e DATAR, 1989; DATAR, KULP, LAMBERT, 2001; FELTHAM e XIE, 1994; HOLMSTROM, 1979).

Outra discussão acerca da aplicabilidade do princípio da controlabilidade concentra-se em sua validade em ambientes que contenham alta incerteza, em que medidas contábeis de desempenho não seriam capazes de viabilizar esse princípio em situações percebidas como incertas, mas apenas quando houver estabilidade ambiental (HARTMANN, 2000).

O princípio da controlabilidade aplicado no desenho de um sistema de contabilidade gerencial indica que as práticas gerenciais adotadas pelas empresas deveriam ser capazes de evitar a responsabilização dos gestores por resultados financeiros que não dependam de seus esforços ou que não reflitam a intensidade desse esforço (COLBERT e SPICER, 1995; INDJEJIKIAN E NANDA, 1999; LAMBERT, 2001; MELUMAD, MOOKHERJEE, REICHELSTEIN, 1992; SPRINKLE, 2003). As principais práticas de contabilidade gerencial associadas com esse princípio são: centros de responsabilidade, custo padrão, preço de transferência, orçamento e medidas de desempenho.

Como resultado final do atendimento ao princípio da controlabilidade, temos o fato de que os gestores somente seriam avaliados e remunerados com base no que eles podem controlar (Figura 1). Significaria dizer que a existência de sistemas de incentivos gerenciais, por meio de remuneração variável, implicaria a presença de práticas associadas ao princípio da controlabilidade para que, desse modo, fosse possível a separação entre os fatores controláveis e incontroláveis. O resultado seria a consequente remuneração gerencial, tendo por base, principalmente, os fatores controláveis.

\section{Sistemas de incentivos gerenciais}

Sistemas de incentivos gerenciais podem ser utilizados como ferramenta de alinhamento de interesses para 
alcance dos objetivos organizacionais (ANTHONY e GOVINDARAJAN, 2008). Especificamente, incentivos monetários podem influenciar o comportamento dos agentes, o que poderá garantir determinado nível de desempenho, sendo geralmente baseados no alcance de metas orçamentárias que refletem suas áreas de responsabilidade (BAILEY, BROWN, COCCO, 1998; BAKER, JENSEN, MURPHY, 1988; MELUMAD, MOOKHERJEE, REICHELSTEIN, 1992).

Assim, sistemas de incentivos monetários parecem estar relacionados a uma série de práticas gerenciais, tais como centros de responsabilidade, preço de transferência, orçamento e avaliação de desempenho (ATKINSON e outros, 1995; BANKER e DATAR, 1989; MERCHANT e VAN DER STEDE, 2003). Entre os tipos de incentivo monetário, destacam-se: salário-base, bônus anuais, planos de incentivo de longo prazo e incentivos baseados em ações (MURPHY, 1999).

O salário-base não depende do nível de desempenho dos gestores, isto é, representa uma remuneração fixa. Os demais tipos de incentivo são dependentes do alcance de um determinado nível de desempenho, geralmente associados às metas orçamentárias (MURPHY, 1999); por isso, são classificados como remuneração variável. A diferença entre eles reside, entre outros aspectos, no horizonte temporal para definição do direito de recebimento da remuneração e no risco associado à capacidade dos gestores de influenciarem as medidas de desempenho associadas a cada tipo de remuneração. Bônus anuais, por exemplo, normalmente envolvem um horizonte de tempo de um ano, enquanto planos de incentivo de longo prazo, incluindo a remuneração baseada em ações, envolvem horizontes de tempo maiores (MERCHANT e VAN DER STEDE, 2003).

No que se refere ao risco, é geralmente admitido que bônus anuais definidos com base no alcance de metas orçamentárias fazem com que os gestores assumam um nível de risco relativamente menor quando comparados com remuneração baseada em ações, tais como opção de ações (MURPHY, 1999). A razão é que o preço de ação é percebido como sendo afetado por outras variáveis, tais como o cenário econômico, além do esforço gerencial, tornando esse indicador mais difícil de ser controlado.

Embora os tipos de incentivos gerenciais oferecidos pelas empresas possam variar entre os diversos níveis hierárquicos, foram considerados neste trabalho somente os incentivos monetários oferecidos aos gestores.

\section{Centros de responsabilidade}

Uma das principais consequências do atendimento ao princípio da controlabilidade está relacionada ao desenho de sistemas de contabilidade por responsabilidade (HIRST, 1983; INDJEJIKIAN e NANDA, 1999; MODELL e LEE, 2001). Por meio dos centros de responsabilidade, determinadas decisões são delegadas aos gestores, estejam elas relacionadas com custos, com receitas ou com investimentos (MELUMAD, MOOKHERJEE, REICHELSTEIN, 1992). Desse modo, os gestores iriam prestar contas apenas de atividades e de recursos associados com o seu centro de responsabilidade (INDJEJIKIAN e NANDA, 1999). Portanto, centros de responsabilidade permitiriam a mensuração, a divulgação e a avaliação do desempenho de gestores ou de uma unidade organizacional em relação apenas a determinadas tarefas e metas organizacionais (COOPER, HAYES, WOLF, 1981; ZIMMERMAN, 2000).

Melumad, Mookherjee e Reichelstein (1992) desenvolvem um modelo de agência por meio do qual avaliam o papel de centros de responsabilidade com apenas um responsável, comparando-o a uma estrutura baseada em times nos quais há, pelo menos, dois responsáveis. O modelo desenvolvido sugere que estruturas de responsabilidade podem funcionar como eficientes instrumentos de incentivos, reduzindo custos de agência, ao permitir que o principal tenha que negociar apenas com o responsável pelo centro de responsabilidade. Esse último, por sua vez, será responsável também pela delegação de atividades a outros agentes.

\section{Figura 1 - Elo entre princípio de controlabilidade e sistemas de incentivo gerencial}

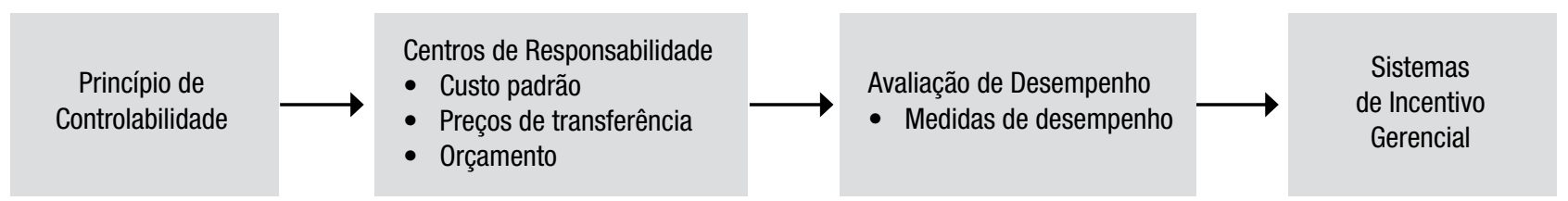


Tal eficiência é considerada maior diante de maiores limitações no processo de comunicação (MELUMAD, MOOKHERJEE, REICHELSTEIN, 1992).

A atribuição de direitos de decisão por meio da contabilidade por responsabilidade representa, assim, uma das principais funções da contabilidade gerencial (ZIMMERMAN, 2000). Desse modo, entende-se que, ao estabelecer a responsabilidade dos gestores apenas pelos fatores associados aos seus respectivos centros de responsabilidade e a principal remuneração gerencial atrelada ao desempenho em relação a tais fatores, tem-se uma aplicação direta do princípio da controlabilidade.

\section{Custo padrão}

Custo padrão representa uma técnica utilizada pela contabilidade gerencial para identificação de erros ou ineficiências no processo de produção, por meio do estabelecimento de padrões que serão utilizados como base para avaliação do desempenho operacional atual (BOYNS e EDWARDS, 2007). O custo padrão representa, assim, metas eficientes e percebidas como possíveis de serem atingidas, sendo estabelecidas no início do processo de produção para os custos das atividades a serem consumidas pelos produtos (ATKINSON e outros, 1995).

No processo de avaliação das variações em relação ao custo padrão, tendo por base o princípio da controlabilidade e a contabilidade por responsabilidade, os livros-textos em contabilidade destacam que os gestores somente seriam responsabilizados por variações que estivessem sob a possibilidade de seu controle (JIAMBALVO, 2001). Por esse motivo, Atkinson e outros (1995) enumeram como uma das limitações referentes ao custo padrão o fato de o método ser de pouca utilidade em ambientes em que a tecnologia de produção não seja estável e com elevado grau de incerteza em relação aos negócios, o que poderia implicar a impossibilidade de atendimento ao princípio da controlabilidade.

Em centros de custos, em que o desempenho é avaliado por meio da análise de variação entre o custo real e o custo padrão, os incentivos gerenciais estariam associados com a magnitude dessa variação. Lin e Yu (2002), por exemplo, desenvolvem um estudo de caso em que descrevem o processo de implementação de um sistema de custo padrão por responsabilidade ao qual está associado um sistema de remuneração para incentivar os gestores. Entende-se, desse modo, que a utilização do custo padrão pode representar uma prática de contabilidade gerencial necessária para que sistemas de incentivo gerencial possam existir de acordo com o princípio da controlabilidade.

\section{Preço de transferência}

Preços de transferência representam uma forma de alocação de recursos dentro das organizações, sendo esses recursos representados por produtos intermediários que são transferidos de uma divisão para outra, dentro de uma mesma empresa (LAMBERT, 2001). Os preços de transferência são, geralmente, definidos dentro de um intervalo entre o custo incremental de produzir o produto intermediário e o preço de mercado desse produto (HORNGREN, DATAR, FOSTER, 2004). Preços de transferência envolvem, geralmente, dois elementos: o primeiro está relacionado à existência ou não de autoridade por parte dos gestores quanto às decisões sobre a aquisição dos produtos intermediários, isto é, se serão adquiridos internamente ou externamente; e o segundo elemento refere-se ao condicionamento da decisão de aquisição interna, relacionado à forma pela qual o produto intermediário será avaliado, ou seja, com base no custo, no mercado ou no valor negociado (MEER-KOOISTRA, 1994).

A literatura contábil menciona que uma das principais vantagens de se utilizarem preços de transferência é a possibilidade de oferecer maior autonomia de decisão aos gestores em relação à sua área de responsabilidade, permitindo a mensuração da contribuição que ele oferece para o desempenho global da empresa (HORNGREN, DATAR, FOSTER, 2004). Preços de transferência podem ser percebidos como essenciais, no processo de avaliação e de mensuração dos sistemas de incentivos gerenciais, para que as divisões com oferta de produtos intermediários funcionem como centros de lucro ou de investimento. Assim, os preços de transferência estariam associados ao princípio da controlabilidade por permitirem que os gestores mensurem os resultados de seus esforços relacionados à sua área de responsabilidade.

Preços de transferência são percebidos como práticas relevantes para o desenho e para a implementação de sistemas de controle e de informação gerencial (COLBERT e SPICER, 1995). Especificamente, pesquisas em contabilidade gerencial têm destacado a importância de se incorporarem sistemas de incentivo gerencial associados aos preços de transferência (CHAN, 1998). É possível, portanto, que a presença de preços de transferência não seja uma condição imprescindível, 
para que sistemas de incentivos gerenciais possam ser desenvolvidos. Especialmente quando esses sistemas forem referentes a divisões que lidam com produtos intermediários e têm o desempenho avaliado com base na contribuição que eles geram para o resultado final da empresa.

\section{Orçamento}

Orçamento pode ser entendido como um plano detalhado de lucros de curto prazo por meio do qual são atribuídas responsabilidades (WELSCH, HILTON, GORDON, 1988). Orçamentos também representam uma forma de alocação de recursos dentro das empresas, sendo que, nesse caso, o recurso é representado pelo capital que é transferido do superior para o subordinado (LAMBERT, 2001). Embora possam ser entendidos como instrumentos de política e poder, tradicionalmente, orçamentos são vistos como instrumentos gerenciais que facilitam e que viabilizam o processo de aquisição e de alocação de recursos entre divisões, sendo a aplicação desses recursos direcionada ao alcance de objetivos organizacionais (COVALESKI e DIRSMITH, 1986).

Orçamento está associado a diversas outras práticas de contabilidade gerencial, tais como: custos, contabilidade por responsabilidade, avaliação de desempenho e remuneração (COVALESKI e outros, 2007). A utilização do orçamento é importante para a criação de centros de responsabilidade que permitam a aplicação do princípio da controlabilidade (BRUNS e WATERHOUSE, 1975; MERCHANT, 1984). Orçamentos são, assim, instrumentos gerenciais que refletiriam as metas de curto prazo de uma divisão e os recursos alocados necessários para a viabilização dessas metas, sendo o desempenho do gestor divisional avaliado, principalmente, em relação ao alcance dos objetivos e à alocação de tais recursos relativos à sua área de responsabilidade.

Orçamentos são elementos importantes para a definição de incentivos. Levantamentos demonstram que bônus anuais baseados em orçamento representam o principal tipo de incentivo usado pelas empresas para remunerar os gestores (MURPHY, 1999). Esquemas de incentivo baseados em orçamento são, de fato, o principal tipo de remuneração explorado por estudos contábeis analíticos (ver, por exemplo, LAMBERT e LARCKER, 1987; FELTHAM e XIE, 1994; DIKOLLI, 2001; DIKOLLI e VAYSMAN, 2006; HEMMER, 1996; SLIWKA, 2002; DUTTA e REICHELSTEIN, 2003). Portanto, a presença de sistemas de incentivo estaria associada ao uso de orçamentos, servindo de base para o acompanhamento do desempenho gerencial.

\section{Avaliação de desempenho}

O sistema de avaliação de desempenho tem como principal objetivo fazer com que os gestores tomem decisões congruentes com as metas organizacionais (ANTHONY e GOVINDARAJAN, 2008). Desse modo, o sistema de avaliação de desempenho parte da estratégia empresarial e inclui elementos tais como: i) as dimensões do desempenho que serão acompanhadas, considerando se serão apenas financeiras ou também não financeiras; ii) as metas a serem alcançadas, inclusive decisões quanto ao grau de participação e de dificuldade de alcance das metas; e iii) a remuneração decorrente do desempenho obtido, em especial, quando envolvendo decisões referentes ao grau de controle que as pessoas possuem sobre as áreas pelas quais elas são responsáveis (FITZGERALD, 2007).

A literatura em contabilidade tem dedicado razoável atenção à identificação das medidas de desempenho que deveriam ser incluídas no plano de remuneração gerencial, bem como ao peso a ser atribuído a cada uma dessas medidas (ver BANKER e DATAR, 1989; BOUWENS E VAN LENT, 2006; DUTTA e REICHELSTEIN, 2003; GIBBS e outros, 2004; FELTHAM e XIE, 1994; MOERS, 2005; MOERS, 2006; VAN RINSUM, 2006). Segundo o princípio da controlabilidade, qualquer indicador de desempenho controlável, desde que não esteja correlacionado a outro já existente, tem conteúdo informacional que justifica sua inclusão no plano de remuneração gerencial (LAMBERT, 2001). Como consequência, pesquisas contábeis baseadas em teoria da agência têm buscado identificar as medidas de desempenho que deveriam ser incluídas no plano de remuneração gerencial de modo a influenciar os gestores a tomarem decisões congruentes com as expectativas organizacionais (INDJEJIKIAN e NANDA, 1999).

Sistemas de avaliação de desempenho e sistemas de remuneração representam as duas dimensões dos planos de remuneração gerencial (BANKER e DATAR, 1989). Em geral, os tipos de incentivo gerencial envolvem alguma medida de desempenho que servirá como base para definição do direito ou não de recebimento de remuneração variável. Bônus anuais, por exemplo, incluem uma ou mais medidas de desempenho para a definição do recebimento de incentivos (MURPHY, 1999). Nesse sentido, é possível esperar que a presença de sistemas de incentivos gerenciais demande um 
processo de avaliação de desempenho para a definição dos gestores que terão direito ao recebimento de remuneração variável.

\section{METODOLOGIA}

Realiza-se um estudo de campo para a coleta de dados por meio de entrevistas com questionário estruturado. A construção e a validação do instrumento de coleta de dados foram feitas com base em questões abertas e fechadas, sendo as fechadas coletivamente exaustivas e mutuamente excludentes, fundamentando-se nos pressupostos estatísticos e utilização de técnicas estatísticas. Assim, para a coleta de dados, foi utilizado questionário contendo 44 questões abertas e fechadas.

As referidas questões foram estruturadas em seis blocos, incluindo questões sobre (i) a empresa do respondente, (ii) o papel da contabilidade gerencial, (iii) as ferramentas de contabilidade gerencial, (iv) os benefícios da implantação das ferramentas, (v) os fatores que restringem ou motivam a implantação das ferramentas na empresa e (vi) os respondentes.

Para os propósitos deste estudo, foi utilizada apenas parte das informações contidas nos blocos i, iii e vi. No que se refere especificamente ao bloco iii, as variáveis independentes - análise por centro de responsabilidade, custo padrão, preço de transferência, orçamento anual e avaliação de desempenho - foram mensuradas utilizando-se uma escala de quatro pontos, incluindo os seguintes itens: amplamente utilizado, em fase de implantação, parcialmente utilizado e não utilizado. Um quinto item, não soube responder, também foi incluído. Para fins de análise de dados, essa escala foi reduzida a uma escala binária, em que as respostas ao item amplamente utilizado representaram a presença das práticas de contabilidade gerencial, enquanto as respostas aos demais itens representaram a ausência de tais práticas. Por sua vez, a variável dependente - sistemas de incentivo gerencial - foi mensurada utilizando-se um escala binária. As questões para o bloco iii foram construídas com base em Soutes e Guerreiro (2007).

\section{Amostra}

Utilizaram-se como população as empresas que compõem o ranking elaborado pela Revista 200 Maiores Empresas do Estado do Espírito Santo, edição 2007, das quais foram coletados dados referentes a 150 empre- sas. A escolha desse grupo de empresas deu-se pela acessibilidade e pela disponibilização dos dados por elas, sendo a amostra não probabilística. Foram levantados dados de empresas que atuavam em diversos setores econômicos, conforme apresentado na Tabela 1 .

\begin{tabular}{l|c|c|}
\hline \multicolumn{3}{|c|}{$\begin{array}{l}\text { Tabela 1 - Atuação das empresas nos } \\
\text { setores econômicos }\end{array}$} \\
\hline \multicolumn{1}{|c|}{ Setor econômico } & Frequência & $\%$ \\
\hline Comercial & 49 & $33 \%$ \\
\hline Indústria de Utilidade Pública & 4 & $3 \%$ \\
\hline Industrial & 45 & $29 \%$ \\
\hline Instituições Financeiras & 4 & $3 \%$ \\
\hline Serviços & 48 & $32 \%$ \\
\hline Total & $\mathbf{1 5 0}$ & $\mathbf{1 0 0} \%$ \\
\hline
\end{tabular}

O instrumento de coleta de dados foi aplicado por meio de entrevista pessoal in loco, permitindo o imediato esclarecimento de eventuais dúvidas dos entrevistados acerca das questões contidas no questionário ou até mesmo dúvidas da própria pesquisa. A aplicação do questionário ocorreu por meio de visitas às empresas, com data e horário agendados previamente por contato telefônico com o respondente, no período de abril a dezembro de 2008. O questionário foi aplicado aos gestores das empresas da amostra ou aos responsáveis pelos processos de tomada de decisões, sendo as principais características relacionadas aos respondentes demonstradas na Tabela 2 .

\section{Modelo e variáveis utilizadas}

Foram utilizados como variável dependente os sistemas de incentivo gerencial que são oferecidos aos gestores pelas empresas, sendo esses incentivos monetários. Assumiu-se valor 1 para as empresas da amostra que oferecem algum sistema de incentivos e valor 0 para as empresas que não oferecem tais sistemas.

Foram utilizadas como variáveis independentes aquelas previstas pelo princípio da controlabilidade e associadas ao fato de a empresa oferecer ou não sistemas de incentivo gerencial aos gestores. São elas: análise por centro de responsabilidade, custo padrão, preço de transferência, orçamento anual e avaliação de desempenho.

Para verificar a associação entre as variáveis previstas pelo princípio da controlabilidade e os sistemas de 
incentivos gerenciais, utilizou-se a ferramenta estatística de regressão logística. Esse método de análise de dados é recomendado em casos nos quais a variável dependente analisada é binária, permitindo que a estimativa de probabilidade de sua ocorrência seja feita com base em um conjunto de variáveis explicativas contínuas ou binárias (CORRAR, PAULO, DIAS FILHO, 2007; HAIR e outros, 2005).

Desse modo, considera-se a regressão logística o método de análise multivariada de dados que apresenta adequação ao objetivo deste estudo, dada a restrição da escala da variável independente. Precisamente, o uso da regressão logística permitirá estimar a probabilidade de existência ou presença de sistemas de incentivo gerencial nas empresas analisadas com base na existência de determinadas práticas de contabilidade gerencial.

O modelo é descrito conforme a Equação 1 abaixo:

Sist. Inc. $=\beta_{1}+\beta_{2}$ Orç $+\beta_{3}$ CustPad $+\beta_{4}$ AnCenResp

$+\beta_{5}$ PreTrans $+\beta_{6}$ ReuAvDes $+\mathrm{e}$

Onde:

- Sist. Inc. - Sistemas de incentivos gerenciais, assumindo valor 0 para as empresas que oferecem remuneração fixa e valor 1 para as que oferecem remuneração variável;

- Orç - Orçamento anual, assumindo valor 1 para as empresas que utilizam orçamento anual e 0 para as empresas que não utilizam;

- CustPad - Custo padrão, assumindo valor 1 para as empresas que utilizam custo padrão e valor 0 para as empresas que não utilizam;

- AnCenResp - Análise por centro de responsabilidade, assumindo valor 1 para as empresas que realizam análise por centro de responsabilidade e valor 0 para as empresas que não realizam;

- PreTrans - Preço de transferência, assumindo valor 1 para as empresas que utilizam preço de transferência interno e valor 0 para as empresas que não utilizam;

- ReuAvDes - Proxy para avaliação de desempenho, assumindo valor 1 para as empresas que realizam reunião para avaliação de desempenho e valor 0 para as empresas que não realizam reunião para avaliação de desempenho.

\section{ANÁLISE DOS RESULTADOS}

A Tabela 3 demonstra a estatística descritiva referente às variáveis da amostra analisada:

A estatística descritiva da amostra é apresentada com base na contagem das respostas dadas pelas empresas quanto à utilização das ferramentas de contabilidade gerencial. Destacam-se como ferramentas mais utilizadas pelas empresas da amostra: reunião para avaliação de desempenho (91\%), sistemas de incentivos gerenciais (62\%), orçamento anual (51\%) e análise por centro de responsabilidade (51\%). As demais ferramentas são utilizadas por menos de $50 \%$ das empresas amostradas, sendo custo padrão a variável que apresentou menor utilização (22\%).

A Tabela 4 apresenta a matriz de correlação entre as variáveis. Pode-se perceber que, entre as práticas de contabilidade gerencial, somente custo padrão não apresenta correlação significante com sistemas de incentivo gerencial. Percebe-se a presença de multicolinearidade entre as variáveis independentes, o que pode afetar as estimativas dos coeficientes da regressão logística. Por fim, percebe-se que tamanho apresenta correlação com análise do centro de responsabilidade e orçamento.

$\mathrm{Na}$ Tabela 5, são demonstrados os resultados encontrados para a Equação 1, como segue:

\section{Tabela 2 - Características dos respondentes}

\begin{tabular}{|c|c|c|c|c|c|c|c|c|c|c|c|c|c|c|c|c|c|}
\hline \multicolumn{7}{|c|}{ Formação acadêmica } & \multicolumn{6}{|c|}{ Cargo ocupado } & \multicolumn{5}{|c|}{ Tempo no cargo ocupado } \\
\hline 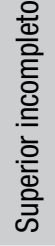 & 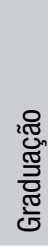 & 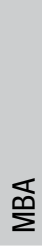 & 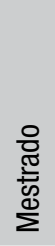 & $\begin{array}{l}\text { 유 } \\
\text { 휴 } \\
\text { 흐 }\end{array}$ & $\begin{array}{l}\stackrel{\infty}{0} \\
\text { 竞 }\end{array}$ & 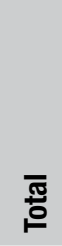 & 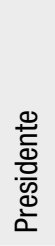 & 离 & 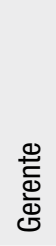 & $\begin{array}{l}\overline{0} \\
\overline{0} \\
\text { 히 } \\
\text { 히 }\end{array}$ & 蒡 & $\begin{array}{l}\overline{\text { ब्ञ }} \\
\text { }\end{array}$ & 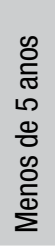 & 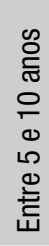 & 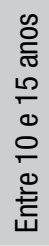 & 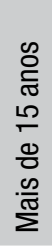 & 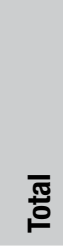 \\
\hline 11 & 87 & 9 & 17 & 2 & 24 & 150 & 24 & 75 & 13 & 2 & 36 & 150 & 77 & 36 & 26 & 11 & 150 \\
\hline
\end{tabular}


O resultado obtido mostra que as associações observadas entre sistemas de incentivos gerenciais e as práticas de contabilidade gerencial não ocorrem ao acaso (Prob $>$ chi2 $=0,0003$ ). Contudo, apenas as variáveis orçamento anual, análise por centro de responsabilidade e reunião para avaliação de desempenho apresentaram significância estatística. Interpretando-se em termos de odds ratios (razão de chances), pode-se dizer que, na amostra, a chance de uma empresa utilizar orçamento anual e possuir sistemas de incentivos é cerca de 2,76 vezes maior do que a de não utilizar ambos. A mesma linha de interpretação aplica-se ao uso da análise por centro de responsabilidade (chance cerca de 2,03 vezes maior) e, especialmente, à reunião para avaliação de desempenho (chance cerca de 3,13 vezes maior). Ao dividir-se a amostra para validação do modelo, percebe-se que o percentual geral de acerto do modelo foi de $67 \%$. Portanto, pode-se concluir que o uso de sistemas de incentivos está associado à utilização dessas três ferramentas gerenciais.

Considerando apenas as práticas de contabilidade gerencial que se mostraram significativamente relevantes, utiliza-se um modelo mais parcimonioso, incluindo-se apenas tais variáveis. Para tanto, foram desprezadas as variáveis custo padrão e preço de transferência, que não se mostraram estatisticamente significantes

\section{Tabela 3 - Matriz de correlação}

\begin{tabular}{|c|c|c|c|c|c|c|c|}
\hline Sist. Inc. & AnCenResp & CustPad & PreTrans & Orç & ReuAvDes & Tam & \\
\hline \multirow[t]{2}{*}{ Sist. Inc. } & \multirow[t]{2}{*}{1,00} & $0,28^{*}$ & 0,05 & $0,15^{\star \star \star}$ & $0,31^{*}$ & $0,22^{*}$ & 0,00 \\
\hline & & 0,0005 & 0,5349 & 0,0753 & 0,0001 & 0,0066 & 0,9818 \\
\hline \multirow[t]{2}{*}{ AnCenResp } & & \multirow[t]{2}{*}{1,00} & 0,10 & $0,32^{*}$ & $0,39^{*}$ & $0,19^{* *}$ & $0,22^{\star \star}$ \\
\hline & & & 0,2303 & 0,0001 & 0,0000 & 0,0186 & 0,0258 \\
\hline \multirow[t]{2}{*}{ CustPad } & & & \multirow[t]{2}{*}{1,00} & 0,03 & $0,23^{*}$ & $0,17^{\star \star}$ & 0,05 \\
\hline & & & & 0,6783 & 0,0051 & 0,0371 & 0,6226 \\
\hline \multirow[t]{2}{*}{ PreTrans } & & & & \multirow[t]{2}{*}{1,00} & $0,15^{\star \star \star}$ & 0,13 & $-0,06$ \\
\hline & & & & & 0,0653 & 0,1138 & 0,5379 \\
\hline \multirow[t]{2}{*}{ Orç } & & & & & \multirow[t]{2}{*}{1,00} & 0,19 & $0,37^{\star}$ \\
\hline & & & & & & 0,1860 & 0,0001 \\
\hline \multirow[t]{2}{*}{ ReuAvDes } & & & & & & 1,00 & 0,07 \\
\hline & & & & & & & 0,4520 \\
\hline Tam & & & & & & & 1,00 \\
\hline
\end{tabular}

0 valor-p é apresentado abaixo de cada coeficiente de correlação.

${ }^{\star},{ }^{\star \star},{ }^{\star \star \star}$ Estatisticamente significantes ao nível de $1 \%, 5 \%$ e $10 \%$ respectivamente.

\section{Tabela 4 - Estatística descritiva dos dados}

\begin{tabular}{|l|c|c|c|c|c|c|}
\hline \multicolumn{1}{|c|}{ Variável } & \multicolumn{2}{|c|}{ Não } & \multicolumn{2}{c|}{ Sim } & \multicolumn{2}{c|}{ Total } \\
\hline & Quantidade & $\%$ & Quantidade & $\%$ & Quantidade \\
\hline Sistemas de incentivos gerenciais & 57 & $38 \%$ & 93 & $62 \%$ & 150 & $100 \%$ \\
\hline Orçamento anual & 73 & $49 \%$ & 77 & $51 \%$ & 150 & $100 \%$ \\
\hline Custo padrão & 117 & $78 \%$ & 33 & $22 \%$ & 150 & $100 \%$ \\
\hline Análise por centro de responsabilidade & 73 & $49 \%$ & 77 & $51 \%$ & 150 & $100 \%$ \\
\hline Preço de transferência interno & 100 & $67 \%$ & 50 & $33 \%$ & 150 & $100 \%$ \\
\hline Reunião avaliação de desempenho & 14 & $9 \%$ & 136 & $91 \%$ & 150 & $100 \%$ \\
\hline
\end{tabular}


(Equação 2):

Sist. Inc. $=\beta_{1}+\beta_{2}$ Orç $+\beta_{3}$ AnCenResp $+\beta_{4}$ ReuAvDes $+\mathrm{e}$

Na Tabela 6, são demonstrados os resultados encontrados na Equação 2, como segue:

No novo modelo, as associações observadas também não ocorrem ao acaso (Prob > chi2 = 0,0000). O Pseudo R2 calculado é muito similar àquele encontrado para o modelo da Equação 1 (0,1146 contra 0,1181). Desse modo, ao serem desprezadas as variáveis não significantes do primeiro modelo, o segundo praticamente nada perde em termos de poder explicativo. Em termos de validação do modelo, o percentual geral de acerto do modelo foi de 68\%.

Em busca de algum outro fator que pudesse influenciar as demais variáveis, incluiu-se na equação o tamanho como variável de controle. O objetivo era o de minimizar o efeito escala, devendo-se, ainda, ao fato de que o tamanho das empresas pode influenciar o seu comportamento (GUPTA, 1980; SUTTON, 1997; HALL, 1987). Utilizou-se como proxy para tamanho das empresas o logaritmo do ativo total do ano de 2006, conforme utilizado em trabalhos anteriores, tais como Gupta (1980) e Hall (1987), que encontraram evidências de que o tamanho da empresa está relacionado com a lucratividade. Foram criadas duas categorias com base nos valores do logaritmo do ativo total, definindo-se o valor 0 quando o logaritmo do ativo total apresentava-se abaixo da mediana e 1 quando acima da mediana. Inicialmente, aplicou-se o controle por tamanho à Equação 1, resultando na seguinte equação de regressão 3:

Sist. Inc. $=\beta_{1}+\beta_{2}$ Orç $+\beta_{3}$ CustPad $+\beta_{4}$ AnCenResp

$+\beta_{5}$ PreTrans $+\beta_{6}$ ReuAvDes $+\beta_{7}$ Tam $+\mathrm{e}$

\section{Onde:}

- Tam - Tamanho das empresas medido pelo logaritmo do ativo total, assumindo valor 1 para

\section{Tabela 5 - Estatística de regressão logística - Equação 1}

\begin{tabular}{|c|c|c|c|}
\hline Sist. incentivos & Odds ratio & Estatistica - Z & P - value \\
\hline Orç & 2,762989 & 2,56 & $0,011^{\star \star}$ \\
\hline CustPad & 0,759600 & $-0,60$ & 0,549 \\
\hline AnCenResp & 2,035484 & 1,77 & $0,076^{\star \star \star}$ \\
\hline PreTrans & 1,278831 & 0,59 & 0,555 \\
\hline ReuAvDes & 3,138957 & 1,74 & $0,082^{\star \star \star}$ \\
\hline $\begin{array}{l}N^{0} \text { observações: } \\
\text { LR chi2 } \\
\text { Prob > chi2 } \\
\text { PseudoR2 }\end{array}$ & & & $\begin{array}{c}150 \\
23,52 \\
0,0003 \\
0,1181\end{array}$ \\
\hline
\end{tabular}

Onde: ${ }^{*}{ }^{* *},{ }^{* \star \star}$ Estatisticamente significantes ao nível de 1\%, 5\% e 10\% respectivamente.

\section{Tabela 6 - Estatística de regressão logística - Equação 2}

\begin{tabular}{|c|c|c|c|}
\hline Sist. incentivos & Odds ratio & Estatistica - Z & P - value \\
\hline Orç & 2,627516 & 2,50 & $0,012^{\star *}$ \\
\hline AnCenResp & 2,169433 & 2,01 & $0,045^{\star \star}$ \\
\hline ReuAvDes & 3,035163 & 1,71 & $0,087^{\star \star \star}$ \\
\hline $\begin{array}{l}\text { No observações: } \\
\text { LR chi2 } \\
\text { Prob > chi2 } \\
\text { PseudoR2 }\end{array}$ & & & $\begin{array}{c}150 \\
22,84 \\
0,0000 \\
0,1146\end{array}$ \\
\hline
\end{tabular}

Onde: ${ }^{\star}{ }^{\star \star},{ }^{* \star \star}$ Estatisticamente significantes ao nível de $1 \%, 5 \%$ e 10\% respectivamente. 
as empresas com logaritmo de ativo acima da mediana e 0 para abaixo da mediana.

$\mathrm{Na}$ Tabela 7, seguem os resultados encontrados para a Equação 3:

Novamente, o modelo como um todo indica que as associações entre a presença de sistemas de incentivo e as práticas de contabilidade gerencial não ocorrem ao acaso (Prob $>$ chi $2=0,0301$ ). Porém, somente a variável orçamento anual mostrou-se estatisticamente significante: a chance de uma empresa com orçamento anual ter sistemas de incentivos é cerca de 3,26 vezes maior do que a de não os ter, apenas reforçando o encontrado anteriormente com respeito a essa associação. Novamente, o percentual geral de acerto do modelo foi de 68\%. Uma possível causa da perda de explicação das variáveis análise por centro de responsabilidade e reunião para avaliação de desempenho é a multicolinearidade existente entre as variáveis incluídas no modelo.

Em seguida, aplicou-se o controle por tamanho à Equação 2, resultando no modelo representado pela Equação 4, conforme segue:

Sist. Inc. $=\beta_{1}+\beta_{2}$ Orç $+\beta_{3}$ AnCenResp $+\beta_{4}$ ReuAvDes

$+\beta_{5}$ Tam $+\mathrm{e}$

Os resultados encontrados são mostrados na Tabela 8:

Reforçando os resultados anteriores, o modelo como um todo indica que as associações entre a presença de sistemas de incentivos e as práticas de contabilidade gerencial não ocorrem ao acaso (Prob > chi2 $=0,0129)$. Em conformidade com o encontrado na análise da Equação 3, somente a variável orçamento anual mostrou-se estatisticamente significante: a chance de

\section{Tabela 7 - Estatística de regressão logística - Equação 3}

\begin{tabular}{|l|c|c|c|}
\hline \multicolumn{1}{|c|}{ Sist. incentivos } & Odds ratio & Estatistica $\mathbf{Z}$ & $\boldsymbol{P}$ - value \\
\hline Orç & 3,266133 & 2,23 & $0,026^{\star *}$ \\
\hline CustPad & 0,569594 & $-1,08$ & 0,281 \\
\hline AnCenResp & 1,671837 & 1,08 & 0,280 \\
\hline PreTrans & 1,113182 & 0,22 & 0,823 \\
\hline ReuAvDes & 3,507896 & 1,60 & 0,110 \\
\hline Tam & 0,541797 & $-1,24$ & 0,213 \\
\hline No observações: & & & 105 \\
\hline LR chi2 & & & 13,96 \\
\hline Prob > chi2 & & & 0,0301 \\
\hline PseudoR2 & & & 0,1008 \\
\hline
\end{tabular}

Onde: ${ }^{*}{ }^{* *},{ }^{* \star *}$ Estatisticamente significantes ao nível de $1 \%, 5 \%$ e $10 \%$ respectivamente.

Tabela 8 - Estatística de regressão logística - Equação 4

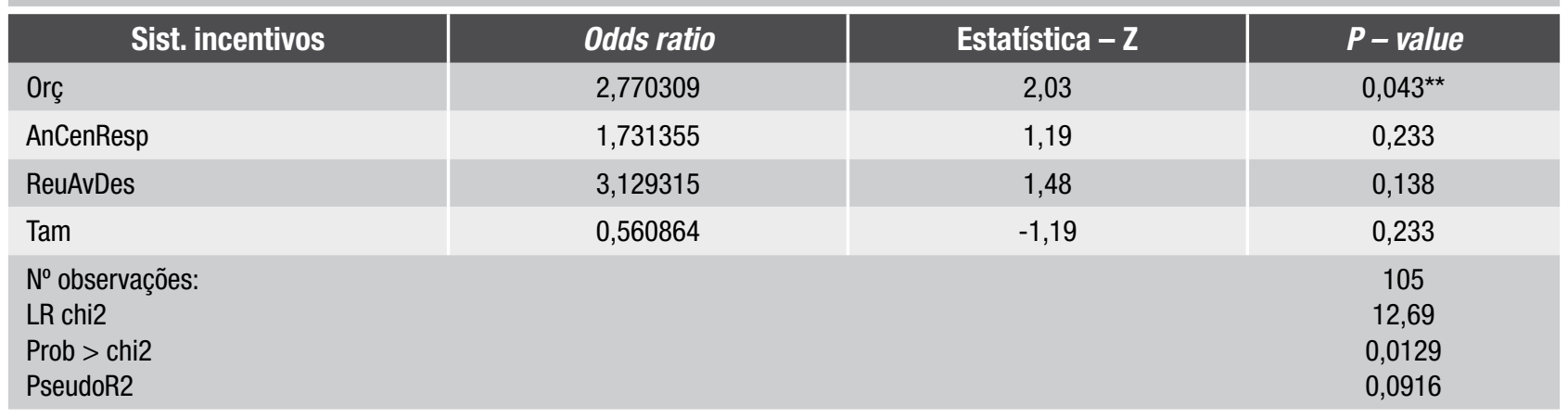

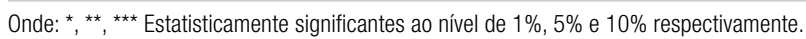


uma empresa com orçamento anual possuir sistemas de incentivos é cerca de 2,77 vezes maior do que a de não os possuir. Para esse último modelo, a divisão da amostra para validação do modelo resultou em um percentual geral de acerto de $70 \%$. Novamente, destaca-se que a perda de explicação das variáveis análise por centro de responsabilidade e reunião para avaliação de desempenho pode ser decorrência da multicolinearidade existente entre as variáveis incluídas no modelo.

\section{CONCLUSÃO E SUGESTÕES PARA FUTURAS PESQUISAS}

Uma das questões centrais associadas ao desenho de sistemas de incentivo gerencial é que os gestores deveriam ser remunerados principalmente em relação ao desempenho obtido em atividades que eles podem controlar. Para tanto, práticas de contabilidade gerencial poderiam ser utilizadas para viabilizar a aplicação do princípio da controlabilidade e, por consequência, permitir que sistemas de incentivo cumpram com seu papel organizacional de influenciar e de motivar o comportamento gerencial.

Este estudo investigou a associação de um conjunto de práticas de contabilidade que viabilizaria a aplicação do princípio da controlabilidade com a presença de sistemas de incentivo gerencial. A pesquisa oferece evidências de que centros de responsabilidade, orçamento e avaliação de desempenho parecem servir de base para a estruturação de tais sistemas de incentivo, não sendo possível dizer o mesmo em relação ao uso de custo padrão e de preço de transferência. Esses resultados indicam que o desenho de sistemas de incentivo gerencial requerem estruturas organizacionais descentralizadas e organizadas em centros de responsabilidade, com delimitada área de responsabilidade gerencial, além de planos de alocação de recursos de curto prazo a serem acompanhados por meio de um processo de avaliação de desempenho. Por outro lado, sistemas de incentivo gerencial parecem não depender de variações entre custo real e custo padrão ou de processos de alocação de recursos por meio de produtos intermediários.

Diversas implicações podem decorrer desses resultados. Em primeiro lugar, na medida em que centros de responsabilidade representam uma prática associada à presença de sistemas de incentivo gerencial, as empresas não parecem dispostas a assumir o custo decorrente de fazer com que os gestores assumam a responsabilidade por muitos fatores incontroláveis, o que pode resultar em áreas residuais de responsabilidades, pelas quais nenhum gestor seria responsável. Tal situação poderia ocorrer, por exemplo, quando uma empresa decide centralizar decisões de investimento de longo prazo, deixando sob responsabilidade dos gestores apenas decisões com efeitos financeiros de curto prazo, relativamente mais controláveis. Uma consequência de tal situação seria a de os gestores ficarem excessivamente focados no curto prazo, isto é, de eles perderem a percepção dos efeitos de longo prazo de suas decisões atuais. Portanto, contribuições adicionais para o entendimento da associação entre centros de responsabilidade e sistemas de incentivo gerencial poderiam ser obtidas de estudos que investiguem o efeito comparativo no desempenho, individual ou organizacional, de empresas que centralizam decisões de investimento de longo prazo e de empresas que incluem essas decisões como parte das responsabilidades gerenciais.

Em segundo lugar, a associação entre orçamento e a presença de sistemas de incentivo gerencial reforça o que a pesquisa em contabilidade gerencial tem identificado, isto é, que a maior parte dos incentivos gerenciais é baseada em orçamento (MURPHY, 1999). Tem sido igualmente percebido por essa literatura que o uso de orçamento para os propósitos de incentivo gerencial pode resultar em comportamentos disfuncionais, tais como: manipulação de resultados, criação de reservas e miopia gerencial (MERCHANT, 1990; VAN DER STEDE, 2000; CHOW, KATO, MERCHANT, 1996). Estudos adicionais poderiam justamente explorar os contextos em que esses comportamentos disfuncionais poderiam ocorrer em decorrência do uso de orçamento para os propósitos de incentivo, comparando, por exemplo, diferentes processos de negociação de metas orçamentárias com diferentes graus de dificuldade e diferentes níveis de rigidez no acompanhamento orçamentário.

Em terceiro lugar, a associação entre avaliação de desempenho e a presença de sistemas de incentivo gerencial representa um dos principais direcionamentos da pesquisa em contabilidade gerencial baseada em teoria da agência (BANKER e DATAR, 1989; FELTHAM e XIE, 1994; DIKOLLI, 2001; DIKOLLI e VAYSMAN, 2006; HEMMER, 1996). Realmente, discutir o efeito de diferentes tipos de incentivo (por exemplo, bônus anuais, opção de ações e planos de incentivo de longo prazo) sobre o comportamento gerencial parece fazer sentido apenas quando se considera o seu efeito interativo com o processo de avaliação de desempenho, em termos 
das medidas de desempenho incluídas no plano de remuneração ou em termos da frequência com que os relatórios de desempenho são avaliados. Pesquisa em contabilidade gerencial poderia, por exemplo, investigar o efeito relativo de diferentes tipos de incentivo em termos de suas propriedades básicas, isto é, medidas de desempenho, período de avaliação e momento de remuneração.

Em quarto lugar, a ausência de associação entre custo padrão e a presença de sistemas de incentivo indica que os gestores não recebem remuneração em decorrência de terem atingido os padrões de produção. Tal fato levanta a questão da utilidade dessa prática de contabilidade gerencial, no sentido de influenciar e de motivar os gestores a atingir os padrões definidos, uma vez que não existem incentivos associados à prática. Pesquisas futuras poderiam investigar a eficiência relativa de sistemas de custeio padrão em contextos em que os gestores recebam incentivos por atingirem os padrões e contextos em que tais incentivos não estejam presentes.

Por fim, a ausência de associação entre preços de transferência e a presença de incentivos gerenciais sugere que o processo de alocação de recursos por meio de produtos intermediários não é utilizado para as finalidades de avaliação e de remuneração de desempenho, que representa um de seus principais benefícios, podendo sua utilidade ser decorrente de questões tributárias. Estudos posteriores poderiam investigar as diferentes finalidades de uso de preços de transferência e o efeito provocado sobre o comportamento dos gestores.

A principal contribuição deste estudo foi a de identificar as práticas de contabilidade gerencial que viabilizam a aplicação do princípio da controlabilidade e que estão associadas à presença de sistemas de incentivo gerencial, sugerindo que a utilização de tais práticas seria uma condição necessária para que a estruturação de sistemas de incentivo que remunerem os gestores, principalmente com base nas atividades que eles podem controlar.

Entretanto, seria preciso considerar que tais práticas de contabilidade gerencial poderiam ser utilizadas para outras finalidades que não as de incentivo gerencial. Centros de responsabilidade, por exemplo, poderiam existir unicamente por questões estruturais de divisão de tarefas e atribuição de responsabilidade, não havendo, realmente, nenhuma vinculação com sistemas de incentivo. De modo semelhante, planos orçamentários poderiam ter como principal finalidade a definição de metas de curto prazo, sem qualquer associação com incentivos gerenciais. Significa dizer que este estudo não está propondo qualquer relação de causalidade, no sentido de que a utilização de uma prática implicará necessariamente a presença de sistemas de incentivo gerencial. Tal conclusão somente poderia ser obtida por meio de outros métodos de pesquisa, tais como experimentos, que permitissem isolar o efeito das variáveis de interesse, ou por meio de outros métodos estatísticos que pudessem explorar relações de causalidade.

Os resultados encontrados precisam ser analisados dentro do seu contexto, sendo que o presente trabalho apresenta algumas limitações, pois existem outros fatores que podem influenciar os resultados encontrados e existem outros tipos de incentivos gerenciais que não foram considerados.

\section{REFERÊNCIAS}

ANTHONY, R. N; GOVINDARAJAN, V. Sistemas de controle gerencial. São Paulo: McGraw-Hill, 2008.

ANTLE, R; DEMSKI, J. S. The controllability principle in responsibility accounting. The Accounting Review, v. 63, n. 4, p. 700-718, 1988.

ATKINSON, A. A; BANKER, R. D; KAPLAN, R. S; YOUNG, S. M. Management accounting. New Jersey: Prentice Hall, 1995.

BAILEY, C. D; BROWN, L. D; COCCO, A. F. The effects of monetary incentives on worker learning and performance in an assembly task. Journal of Management Accounting Research, v. 10, p. 119-131, 1998.

BAKER, G. P; JENSEN, M. C; MURPHY, K. J. Compensation and incentives: practice vs. theory. The Journal of Finance, v. 3, n. 43, p. 593-616, 1988.

BANKER, R. D; DATAR, S. M. Sensitivity, precision, and linear aggregation of accounting signals. Journal of Accounting Research, v. 27, n. 1, p. 21-39, 1989.

BONNER, S. E; SPRINKLE, G. B. The effects of monetary incentives on effort and task performance: theories, evidence, and a framework for research. Accounting, Organizations and Society, v. 27, n. 4-5, p. 303-345, 2002.

BOUWENS, J; VAN LENT, L. Performance measure properties and the effect of incentive contracts. Journal of Management Accounting Research. v. 18, p. 55-75, 2006. 
BOYNS, T; EDWARDS, J. R. The development of cost and management accounting in Britain. In: CHAPMAN, C. S; HOPWOOD, A. G; SHIELDS, M. D. (Eds). Handbook of management accounting research. Oxford: Elsevier, 2007. v. 2, p. 969-1034.

BRUNS Jr, W. J; WATERHOUSE, J. H. Budgetary control and organization structure. Journal of Accounting Research, v. 13, n. 2, p. 177-203, 1975.

CHAN, C. W. Transfer pricing negotiation outcomes and the impact of negotiator mixed-motives and culture: empirical evidence from the U.S. and Australia. Management Accounting Research, v. 9, n. 2, p. 139161, 1998.

CHOW, C. W; KATO, Y; MERCHANT, K. A. The use of organizational controls and their effects on data manipulation and management myopia: a Japan vs U.S. comparison. Accounting, Organizations and Society. v. 21, n. 2-3, p. 175-192, 1996.

COLBERT, G. J; SPICER, B. H. A multi-case investigations of a theory of the transfer pricing process. Accounting, Organizations and Society, v. 20, n. 6, p. 423-456, 1995.

COOPER, D. J; HAYES, D; WOLF, F. Accounting in organized anarchies: understanding and designing accounting systems in ambiguous situations. Accounting, Organizations and Society, v. 6, n. 2, p. 175-191, 1981.

CORRAR, L. J; PAUlO, E; DIAS FILHO, J. M Análise multivariada: para os cursos de administração, ciências contábeis e economia. São Paulo: Atlas, 2007.

COVALESKI, M. A; DIRSMITH, M. W. The budgetary process of power and politics. Accounting, Organizations and Society, v. 11, n. 3, p. 193- 214, 1986.

COVALESKI, M; EVANS III, J. H; LUFT, J; SHIELDS, M. D. Budgeting research: three theoretical perspectives and criteria for selective integration. In: CHAPMAN, C. S; HOPWOOD, A. G; SHIELDS, M. D. (Eds). Handbook of management accounting research. Oxford: Elsevier, 2007. v. 1, p. 587-624.

DATAR, S; KULP, S. C; LAMBERT, R. A. Balancing performance measures. Journal of Accounting Research, v. 39, n. 1, p. 75-92, 2001.
DIKOLLI, S. S. Agent employment horizons and contracting demand for forward-looking performance measures. Journal of Accounting Research, v. 39, n. 3, p. 481-493, 2001.

DIKOLLI, S. S; VAYSMAN, I. Contracting on the stock price and forward-looking performance. European Accounting Review, v. 15, n. 4, p. 445-464, 2006.

DUTTA, S; REICHELSTEIN, S. Leading indicator variables, performance measurement, and long-term versus short-term contracts. Journal of Accounting Research, v. 41, n. 5, p. 837-866, 2003.

FELTHAM, G. A; XIE, J. Performance measure congruity and diversity in multi-task principal/agent relations. The Accounting Review, v. 69, n. 3, p. 429-453, 1994.

FITZGERALD, L. Performance measurement. In: HOPPER, T; NORTHCOTT, D; SCAPENS, R. Issues in management accounting. 3. ed. Harlow: Pearson Education Ltd, 2007. p. $223-241$.

GIBBS, M; MERCHANT, K. A; VAN DER STEDE, W. A; VARGUS, M. E. Determinants and effects of subjectivity in incentives. The Accounting Review, v. 79, n. 2, p. 409-436, 2004.

GUPTA, N. Some alternative definitions of size. The academy of management journal, v. 23, n. 4, p. 759-766, 1980.

HALL, B. H. The relationship between firm size and fir growth in the U.S. manufacturing sector. The Journal of Industrial Economics, v. 35, n. 4, p. 583-606, 1987.

HARTMANN, F. G. H. The appropriateness of RAPM: toward the further development of theory. Accounting, Organizations and Society, v. 25, n. 4-5, p. 451-482, 2000.

HEMMER, T. On the design and choice of modern management accounting measures. Journal of Management Accounting Research, v. 8, p. 87-116, 1996.

HIRST, M. The controllability of financial outcomes. Abacus, v. 19, n. 1, p. 29-38, 1983.

HOLMSTROM, B. Moral hazard and observability. The Bell Journal of Economics, v. 10, n. 1, p. 74-91, 1979.

HORNGREN, C. T; DATAR, S. M; FOSTER, G. Contabilidade de custos. 11. ed. São Paulo: Prentice Hall, 2004. v. 1. 
INDJEJIKIAN, R; NANDA, D. Dynamic incentives and responsibility accounting. Journal of Accounting and Economics, v. 27, n. 2, p. 177-201, 1999.

JIAMBALVO, J. Contabilidade gerencial. Rio de Janeiro: LTC, 2001.

LAMBERT, R. A. Contracting theory and accounting. Journal of Accounting and Economics, v. 32, n. 1-3, p. 3-87, 2001.

LAMBERT, R. A; LARCKER, D. F. An analysis of the use of accounting and market measures of performance in executive compensation contracts. Journal of Accounting Research, v. 25, n. 1, p. 85-125, 1987.

LIN, Z. J; YU, Z. Responsibility cost control system in China: a case of management accounting application. Management Accounting Research, v. 13, n. 4, p. 447-467, 2002.

MEER-KOOISTRA, J. V. D. The coordination of internal transactions: the functioning of ansfer pricing systems in the organizations context. Management Accounting Research, v. 5, n. 2, p. 123-152, 1994.

MELUMAD, N; MOOKHERJEE, D; REICHELSTEIN, S. A theory of responsibility centers. Journal of Accounting and Economics, v. 15, n. 4, p. 445-484, 1992.

MERCHANT, K. A. Influences on departmental budgeting: an empirical examination of a contingency model. Accounting, Organizations and Society, v. 9, n. 3-4, p. 291-307, 1984.

MERCHANT, K. A. The effects of financial controls on data manipulation and management myopia. Accounting, Organizations and Society, v. 15, n. 4, p. 297-313, 1990.

MERCHANT, K. A; VAN DER STEDE, W. A. Management control systems: performance, measurement, evaluation and incentives. Harlow: Prentice Hall, 2003.

MODELL, S; LEE, A. Decentralization and reliance on the controllability principle in the public sector. Financial, Accountability and Management, v. 17, n. 3, p. 191-218, 2001.

MOERS, F. Discretion and bias in performance evaluation: the impact of diversity and subjectivity. Accounting, Organizations and Society, v. 30, n. 1, p. 67-80, 2005.

MOERS, F. Performance measure properties and delegation. The Accounting Review, v. 81, n. 4, p. 897-924, 2006.
MURPHY, K. Executive compensation. In: Ashenfelter, O; Card, D. (Eds). Handbook of labor economics. North Holland: Elsevier, 1999. p. 2485-2563.

SIMONS, R. Revisiting the controllability principle in the twenty-first century. Working Paper, Graduate School of Business Administration Harvard University, 2007. Disponível em: http://www.ssrn.com/. Acesso em 12.3.2009.

SLIWKA, D. On the use of nonfinancial performance measures in management compensation. Journal of Economics E Management Strategy, v. 11, n. 3, p. 487-511, 2002.

SOUTES, D. O; GUERREIRO, R. Estágios evolutivos da contabilidade gerencial em empresas brasileiras. IN: ENCONTRO NACIONAL DA ASSOCIAÇÃO NACIONAL DE PÓS-GRADUAÇÃO EM ADMINISTRAÇÃO, 31, 2007, Rio de Janeiro. Anais. Rio de Janeiro: ANPAD, 2007.

SPRINKLE, G. B. Perspectives on experimental research in managerial accounting. Accounting, Organizations and Society, v. 28, n. 2-3, p. 287-318, 2003.

SUTTON, J. Gibrat's legacy. Journal of Economic Literature, v. 35, n. 1, p. 40-59, 1997.

VAN DER STEDE, W. A. The relationship between two consequences of budgetary controls: budgetary slack creation and managerial short-term orientation. Accounting, Organizations and Society, v. 25, n. 6, p. 609-622, 2000.

VAN RINSUM, M. Performance measurement and managerial time orientation. Rotterdam: ERIM Ph.D. Series Research in Management, 2006.

WELSCH, G. A; HILTON, R; GORDON, P. Budgeting: profit planning and control. 5. ed. Prentice-Hall: New Jersey, 1988.

ZIMMERMAN, J. L. Accounting for decision making and control. 3. ed. New York: McGraw Hill, 2000. 